\title{
Astrocitoma pilomixoide de fossa posterior em criança: relato de caso
}

\author{
Eduardo Nogueira Lima Sousa ${ }^{1}$, João Antonio Pinheiro Marques², Túlio \\ Cícero Spinola Almeida ${ }^{2}$, luri Araujo Honcy ${ }^{3}$, Gilnard Caminha de Menezes \\ Aguiar ${ }^{3}$, Rafael Figueirêdo Pontes ${ }^{3}$, Carlos Vinícius Mota de Melo 4 \\ Faculdade de Medicina da Universidade de Fortaleza, Ceará, Brasil. Instituto Dr. José Frota, Fortaleza, Ceará, Brasil.
}

\section{RESUMO}

Descrever um caso de astrocitoma pilomixoide, um tumor do sistema nervoso central raro, descrito e classificado recentemente diante de sintomatologia e achados pouco específicos. Análise e descrição de um caso de astrocitoma pilomixoide submetido a tratamento cirúrgico no Instituto José Frota, Fortaleza, Ceará. Paciente evolui com regressão importante dos sintomas, sem indícios de recidiva tumoral. O diagnóstico e a classificação correta dos tumores cerebrais são de extrema importância clínica, pois modificam de forma impactante o prognóstico, assim como a abordagem terapêutica.

\section{PALAVRAS-CHAVE}

Astrocitoma, neoplasias encefálicas, sistema nervoso central.

\section{ABSTRACT}

Pilomyxoid astrocytoma of the posterior fossa in children: case report

To describe a pilomyxoid astrocytoma case, a tumor of the central nervous system rare recently described and classified in the face of a specific symptomatology and little found. Analysis and description of a case of pilomyxoid astrocytoma underwent surgical treatment at the José Frota Institute in Fortaleza, Ceará. Patient developed a significant decrease of symptoms without evidence of tumor relapse. The diagnosis and the correct classification of cerebral tumors are of extreme clinical importance, because it changes so as impacting the prognosis as well as therapeutic approach.

\section{KEYWORDS}

Astrocytoma, brain neoplasms, central nervous system.

\section{Introdução}

O astrocitoma pilomixoide (APM) é um tumor raro do sistema nervoso central (SNC), de acometimento preferencial pediátrico, sem predominância por sexo, ${ }^{1}$ que foi classificado em 2007 pela Organização Mundial de Saúde (OMS) como uma variante do astrocitoma pilocítico (AP), com um prognóstico pior e comportamento mais agressivo. ${ }^{2-4} \mathrm{O}$ tumor acomete preferencialmente a região hipotálamo-quiasmática, mas pode se manifestar em qualquer localização do neuroeixo. ${ }^{2,5,6}$

As manifestações clínicas variam de acordo com o tamanho, a localização, a taxa de progressão do tumor, tais como sintomas visuais, distúrbios endócrinos e atraso do desenvolvimento, podendo apresentar sintomas de hipertensão intracraniana..$^{1,4,5,7} \mathrm{O}$ diagnóstico definitivo se dá por meio da análise histopatológica. ${ }^{2,5}$
Não existe consenso em relação à terapêutica definitiva, sendo a ressecção cirúrgica a que se apresenta com resultados favoráveis. ${ }^{1}$

\section{Relato do caso}

Paciente, 8 anos, sexo feminino, natural e procedente de Itapajé, Ceará. Paciente foi referenciada ao serviço de neurocirurgia com quadro de cefaleia progressiva de forte intensidade, associada a vômitos e desequilíbrio postural, com quedas frequentes da própria altura, de início há um mês da data de admissão. Ao exame neurológico, a paciente apresentava ataxia da marcha e estrabismo convergente, sem outras alterações. Foi

\footnotetext{
Acadêmico de Medicina da Universidade de Fortaleza, Fortaleza, Ceará, Brasil.

Neurocirurgião, preceptor da Residência de Neurocirurgia do Instituto Dr. José Frota, Fortaleza, Ceará, Brasil.

Residente de Neurocirurgia do Instituto Dr. José Frota, Fortaleza, Ceará, Brasil.

4 Neurocirurgião, chefe do Serviço de Neurocirurgia do Instituto Dr. José Frota, Fortaleza, Ceará, Brasil.
} 
realizada tomografia de crânio (TC), que evidenciou lesão expansiva em topografia do quarto ventrículo. A paciente foi internada e começou a apresentar diplopia. Realizou-se uma ressonância nuclear magnética (RNM), que apresentou lesão expansiva sólido-cística no interior do quarto ventrículo com realce heterogêneo pelo meio de contraste, sem restrição à difusão, com dimensões de $4 \times 2,5 \times 2,2 \mathrm{~cm}$, determinando uma hidrocelalia significativa (Figuras 1 a 4 ).

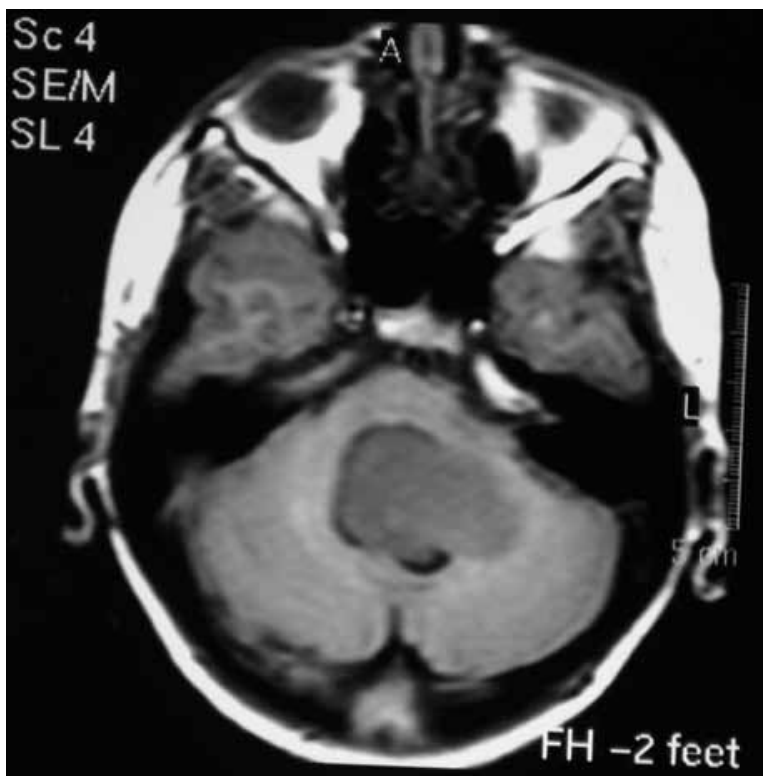

Figura 1 - RNM: corte axial T1, sem contraste, com lesão hipointensa com aparente origem no pedúnculo cerebelar médio esquerdo, com componente exofítico projetando-se medialmente, ocupando o IV ventrículo.

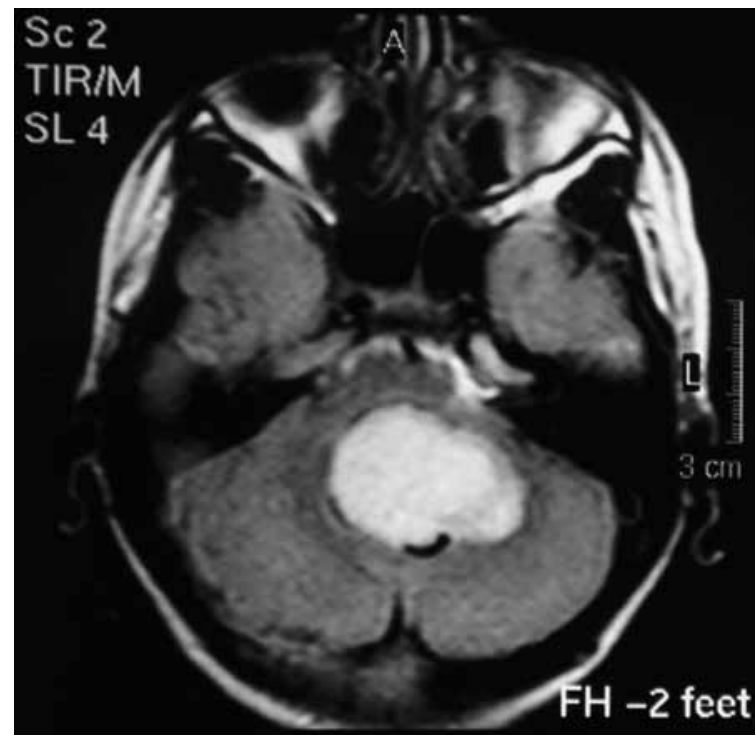

Figura 2 - RNM: corte axial FLAIR com hipersinal intenso na lesão, relacionando-se a um alto teor hídrico e a uma consistência mais amolecida.
A paciente evoluiu com piora do quadro neurológico decorrente da hidrocefalia, submetendo-se a uma derivação ventrículo-peritoneal, com relativa melhora. Submeteu-se a uma ressecção cirúrgica, onde se propôs a retirar o máximo da lesão tumoral. Esta foi diagnosticada como astrocitoma pilomixoide na análise anatomopatológica.

Paciente evoluiu após a cirurgia com nistagmo e desvio do olhar conjugado vertical. Houve melhora gradual dos sintomas, sem déficit motor grosseiro. Atualmente, encontra-se em acompanhamento ambulatorial, sem indícios de progressão tumoral.

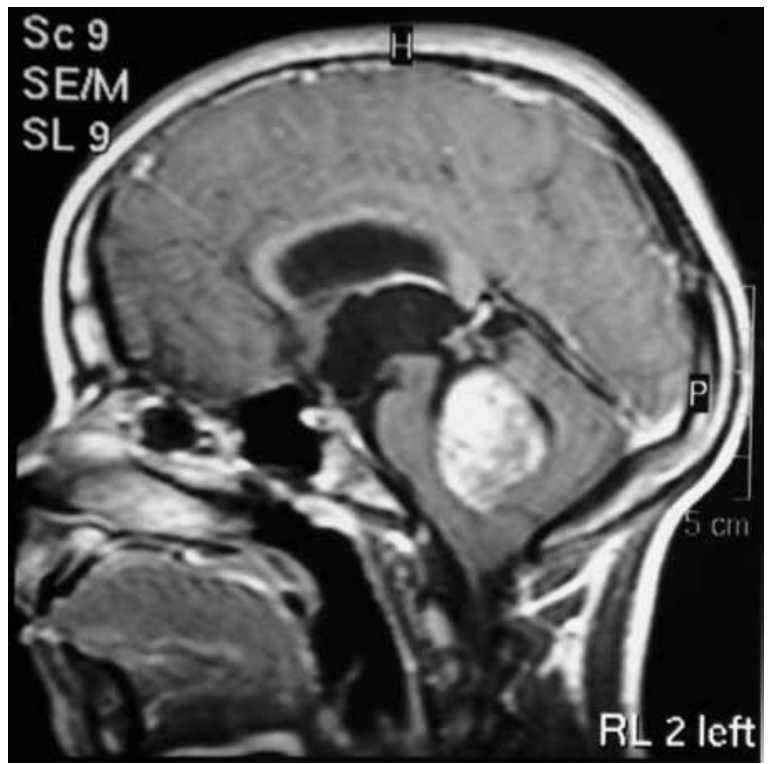

Figura 3 - RNM: corte sagital T1 com contraste, demonstrando realce moderado e heterogêneo, com limites precisos no componente ventricular, evidenciados pela rima liquórica.

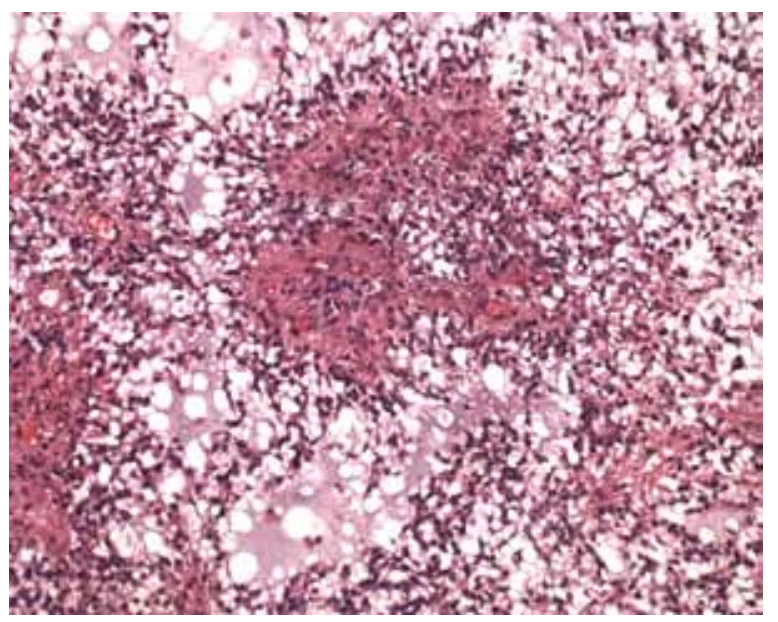

Figura 4 - Aumento de $100 x$ - neoplasia astrocitária com rico estroma mixoide e característico arranjo perivascular. 


\section{Discussão}

Os tumores primários do SNC são relativamente comuns, e na faixa pediátrica representam a segunda neoplasia mais comum. Os tumores apresentam grandes variedades em relação a sua conformação celular, e os sintomas clínicos a eles relacionados são variados, apresentando íntima relação com a localização, tamanho e razão de crescimento; as metástases são relativamente raras. $^{8}$

Os tumores intracranianos são classificados de acordo com o tipo de célula tumoral e o grau histológico; em alguns casos, sua localização e a propagação metastática pelo líquido cefalorraquidiano também são utilizadas. Os tumores gliais compreendem de 50\%$60 \%$ dos tumores primários do SNC; desses, dentre os neuroepiteliais, os astrocitomas são os mais frequentes, constituindo $75 \%$ dos gliomas do encéfalo. ${ }^{8}$

Os astrocitomas podem ser graduados de acordo com seu poder de agressividade, numa escala de I a IV, em que o grau I é benigno, o grau II, por sua vez, apresenta baixa taxa de malignidade, o grau III, também conhecido como anaplásico, é considerado maligno e o grau IV é altamente maligno. ${ }^{8}$

Descritos originalmente em 1985 como astrocitoma pilocítico diencefálico com manifestações clínicas na infância, em 1999 foi descoberta uma entidade distinta do astrocitoma pilocítico por Tihan et al., ${ }^{9}$ que definiu suas características histopatológicas. A OMS modificou a classificação dos tumores do SNC em 2007, sendo o astrocitoma pilomixoide (grau II) uma variante do astrocitoma pilocítico; sua forma variante se comportava de forma mais agressiva, com maiores taxas de metástases pelo SNC e pior prognóstico, se comparada ao astrocitoma pilocítico., , 3,5,7 $^{-}$

O APM é mais comum na faixa pediátrica, sendo a idade média do diagnóstico em torno de 18 meses, embora possa ocorrer em quaisquer faixas etárias, sendo muito raro seu aparecimento em adultos. ${ }^{2-5,10}$ Parece não haver predileção entre os sexos. ${ }^{1} \mathrm{O}$ APM pode ocorrer em qualquer lugar no neuroeixo, mas esses tumores acometem preferencialmente a porção hipotálamo-quiasmática, havendo relatos de casos em fossa posterior, diencéfalo e medula espinhal. ${ }^{2-4,6}$

As manifestações clínicas são bem variadas e inespecíficas e dependem da localização onde se encontra o tumor. Se em região hipotálamo-quiasmática, pode se revelar como anomalia do nervo oculomotor, desregulação endócrina, atraso do desenvolvimento, hidrocefalia e sinais de hipertensão intracraniana. Se localizado em fossa posterior, pode-se manifestar como hidrocefalia ou disfunção cerebelar. De forma geral, os pacientes apresentam atraso do desenvolvimento, alterações sensoriais, vômitos, fraqueza generalizada, associados a disfunções endócrinas e sintomas neurológicos focais e ou visuais. ${ }^{4,5,7} \mathrm{Um}$ aumento da cabeça pode ser o primeiro sintoma em pacientes menores de 1 ano. ${ }^{1}$

Existem fontes literárias que apontam maior incidência do APM em pacientes com neurofibromatose do tipo I, fortalecendo a hipótese de sua origem genética ou congênita, advindo possivelmente da mutação de um único gene; mas permanece incerta tal relação. ${ }^{3,4,6}$

$\mathrm{Na}$ TC e na RNM, comumente se apresentam como uma massa sólida $(84,6 \%),{ }^{4}$ bem circunscrita, homogênea, geralmente na região suprasselar, podendo haver associação com hidrocefalia obstrutiva ou efeito de massa e, menos comumente, um componente cístico, calcificado ou de necrose, o que difere do craniofaringioma, que é comumente calcificado e parcialmente cístico. Em T1 é uma imagem isointensa para parênquima cerebral; em T2 é hiperintensa sem sinais de infiltração do parênquima adjacente. ${ }^{1,3-5,7} \mathrm{Na}$ espectroscopia apresenta baixas concentrações de $\mathrm{N}$-acetil-aspartato, creatina e colina, enquanto o pilocítico apresenta colina aumentada e os outros dois metabólitos diminuídos. Atualmente, é um importante marcador de diferenciação entre os dois tipos histológicos. ${ }^{1,3,7}$

Ao histopatológico, a lesão apresenta um padrão de células pilomixoides neoplásicas monomórfico, composto por uma camada de células bipolares (piloides), em uma emaranhada matriz mixoide. Apresenta ainda um padrão angiocêntrico, com as células tumorais organizadas radialmente em torno dos vasos, em um padrão semelhante a rosetas perivasculares visto nos ependimomas. É caracterizado pela ausência de corpos eosinofílicos granulares e a ausência de fibras de Rosenthal, características que o diferem do AP. Pode apresentar mitoses ocasionais. ${ }^{2-5,7}$

Em relação à terapêutica, não há consenso no que tange ao tratamento definitivo, sendo a ressecção total um indicador confiável de resultados favoráveis. ${ }^{1}$ Em muitas situações, essa ressecção total é difícil, principalmente pelo fato de a maior parte desses tumores se localizar na área hipotálamo-quiasmática. ${ }^{1,4,7}$ A terapia adjuvante é utilizada nos casos de recorrência após a cirurgia, crescimento do tumor ou persistência de sintomas focais após a ressecção. ${ }^{1,4}$ Nos pacientes que são submetidos à ressecção parcial, muitos apresentarão sintomas neurológicos, sendo a quimioterapia o tratamento de escolha em pacientes com menos de 3-5 anos. Acima dessa idade, utiliza-se a combinação de radioterapia e quimioterapia, com melhores resultados, embora haja relatos de má resposta a essa combinação. Em casos disseminados, há relato de resposta favorável com terapêutica clínica com o uso de inibidores da tirosina quinase. ${ }^{1}$

O APM é um tumor raro do SNC, predominante da faixa pediátrica, sendo descrito recentemente como uma 
variante do $\mathrm{AP}^{2,3,10} \mathrm{O}$ tumor apresenta um componente agressivo, assim como um quadro clínico inespecífico; logo, é imperativo um correto diagnóstico, por modificar o tratamento e o prognóstico. ${ }^{2,7,10} \mathrm{O}$ tratamento é essencialmente cirúrgico, com o uso de terapia adjuvante em casos selecionados. ${ }^{1}$

\section{Referências}

1. Domínguez-Páez $M$, Weil-Lara $B$, Rodríguez-Barceló $S$, Medina-Imbroda JM, Puch-Ramírez M, Ros-López B, et al. [Pilomyxoid astrocytoma. Three cases and review]. Neurocirugia (Astur). 2010;21(1):22-9.

2. Altamirano $\mathrm{E}$, Jones $\mathrm{M}$, Drut R. Astrocitoma pilomixoide. Patolog Rev latinoamericana. 2011;49(Supl. 1):S1-4.

3. León MIM. Astrocitoma pilomixoide, un tumor raro de reciente clasificación. In: León MIM, Muñoz IA, Pino MAL, Lara BW, Vázquez E, Riz LC, editores. Arquivo do $29^{\circ}$ Congresso Nacional de la SERAM. Sevilla, Mayo, 2008.

4. Mahore A, Kammar A, Dange N, Epari S, Goel A. Diencephalic juvenile pilomyxoid astrocytoma with leptomeningeal dissemination. Turk Neurosurg. 2011;21(2):222-5.
5. Azad S, Kudesia S, Chawla N, Azad R, Singhal M, Rai SM, et al. Pilomyxoid astrocytoma. Indian J Pathol Microbiol. 2010;53(2):294-6.

6. Linscott LL, Osborn AG, Blaser S, Castillo M, Hewlett $\mathrm{RH}$, Wieselthaler $\mathrm{N}$, et al. Pilomyxoid astrocytoma: expanding the imaging spectrum. AJNR Am J Neuroradiol. 2008;29(10):1861-6.

7. Komotar RJ, Mocco J, Carson BS, Sughrue ME, Zacharia BE, Sisti AC, et al. Pilomyxoid astrocytoma: a review. Med Gen Med. 2004;6(4):42.

8. Braga JL. Astrocitoma anaplásico. Rev Cient HCE. 2008;3(2):82-90.

9. Tihan T, Fisher PG, Kepner JL, Godfraind C, McComb $\mathrm{RD}$, Goldthwaite PT, et al. Pediatric astrocytomas with monomorphous pilomyxoid features and a less favorable outcome. J Neuropathol Exp Neurol. 1999;58:1061-8.

10. Komotar RJ, Burger PC, Carson BS, Brem H, Olivi A, Goldthwaite PT, et al. Pilocytic and pilomyxoid hypothalamic/chiasmatic astrocytomas. Neurosurgery. 2004;54(1):72-9.

\section{Endereço para correspondência}

Eduardo Nogueira Lima Sousa

Rua Doutor Gilberto Studart, 2055, ap. 403

60192-115 - Fortaleza, Ceará

Telefone: (85) 9662-9939

E-mail: eusoueduardo@hotmail.com 